\title{
Enhancement of conformational B-cell epitope prediction using CluSMOTE
}

\author{
Binti Solihah ${ }^{\text {Corresp., } 1,2}$, Azhari Azhari ${ }^{1}$, Aina Musdholifah ${ }^{1}$ \\ ${ }^{1}$ Department of Computer Science and Electronics, Faculty of Mathematics and Natural Sciences, Universitas Gadjah Mada, Yogyakarta, Indonesia \\ 2 Department of Informatics Engineering, Universitas Trisakti, Grogol, Jakarta Barat, Indonesia \\ Corresponding Author: Binti Solihah \\ Email address: binti.solihah@mail.ugm.ac.id
}

Background . A conformational B-cell epitope is one of the main components of vaccine design.It contains separate segments in its sequence, which are spatially close in the antigen chain. The availability of Ag-Ab complex data on Protein Data Bank allows for the development predictive methods. Several epitope prediction models also have been developed, including learning-based methods. However, the performance of the model is still not optimum. The main problem in learning-based prediction models is class imbalance.

Methods. This study proposes CluSMOTE, which is a combination of a cluster-based undersampling method and Synthetic Minority Oversampling Technique. The approach is used to generate other sample data to ensure that the dataset of the conformational epitope is balanced. The Hierarchical DBSCAN algorithm is performed to identify the cluster in the majority class. Some of the randomly selected data is taken from each cluster, considering the oversampling degree, and combined with the minority class data. The balance data is utilized as the training dataset to develop a conformational epitope prediction. Furthermore, two binary classification methods, precisely Support Vector Machine and Decision Tree, are separately used to develop model prediction and to evaluate the performance of CluSMOTE in predicting conformational $b$ cell epitope. The experiment is focused on determining the best parameter for optimal CluSMOTE. Two independent datasets are used to compare the proposed prediction model with state of the art methods. The first and the second datasets represent the general protein and the glycoprotein antigens respectively.

Result. The experimental result shows that CluSMOTE Decision Tree outperformed the Support Vector Machine in terms of AUC and Gmean as performance measurements. The mean AUC of CluSMOTE Decision Tree in the Kringelum and the SEPPA 3 test sets are 0.83 and 0.766 , respectively. This shows that CluSMOTE Decision Tree is better than other methods in the general protein antigen, though comparable with SEPPA 3 in the glycoprotein antigen. 


\section{Enhancement of Conformational B-cell Epitope 2 Prediction using CluSMOTE}

3

4

5

6

7

8

9

10

11

12

13

14

15

16

17

18

19

20

21

22

23

24

25

26

27

28

29

30

31

32

33

34

35

36

37

Binti Solihah $^{1,2}$, Azhari Azhari ${ }^{1}$, Aina Musdholifah ${ }^{1}$

${ }^{1}$ Department of Computer Science and Electronics, Faculty of Mathematics and Natural Sciences, Universitas Gadjah Mada, Yogyakarta, Indonesia

${ }^{2}$ Department of Informatics Engineering, Universitas Trisakti, Jakarta Barat, Indonesia

Corresponding Author:

Binti Solihah ${ }^{1}$

Jl Kyai Tapa No 1, Grogol, Jakarta Barat, 11440, Indonesia

Email address: binti.solihah@mail.ugm.ac.id/binti@trisakti.ac.id

\section{Abstract}

Background. A conformational B-cell epitope is one of the main components of vaccine design. It contains separate segments in its sequence, which are spatially close in the antigen chain. The availability of Ag-Ab complex data on Protein Data Bank allows for the development predictive methods. Several epitope prediction models also have been developed, including learning-based methods. However, the performance of the model is still not optimum. The main problem in learning-based prediction models is class imbalance.

Methods. This study proposes CluSMOTE, which is a combination of a cluster-based undersampling method and Synthetic Minority Over-sampling Technique. The approach is used to generate other sample data to ensure that the dataset of the conformational epitope is balanced. The Hierarchical DBSCAN algorithm is performed to identify the cluster in the majority class. Some of the randomly selected data is taken from each cluster, considering the oversampling degree, and combined with the minority class data. The balance data is utilized as the training dataset to develop a conformational epitope prediction. Furthermore, two binary classification methods, precisely Support Vector Machine and Decision Tree, are separately used to develop model prediction and to evaluate the performance of CluSMOTE in predicting conformational $b$ cell epitope. The experiment is focused on determining the best parameter for optimal CluSMOTE. Two independent datasets are used to compare the proposed prediction model with state of the art methods. The first and the second datasets represent the general protein and the glycoprotein antigens respectively.

Result. The experimental result shows that CluSMOTE Decision Tree outperformed the Support Vector Machine in terms of AUC and Gmean as performance measurements. The mean AUC of CluSMOTE Decision Tree in the Kringelum and the SEPPA 3 test sets are 0.83 and 0.766, respectively. This shows that CluSMOTE Decision Tree is better than other 
38

39

40

41

42

43

44

45

46

47

48

49

50

51

52

53

54

55

56

57

58

59

60

61

62

63

64

65

66

67

68

69

70

71

72

73

74

methods in the general protein antigen, though comparable with SEPPA 3 in the glycoprotein antigen.

\section{Introduction}

A B cell epitope is among the main components of peptide-based vaccines (Andersen et al., 2006; Zhang et al., 2011; Ren et al., 2015). It can be utilized in immunodetection or immunotherapy to induce an immune response (Rubinstein et al., 2008). Many B cell epitopes are conformational and originate from separate segments of an antigen sequence, forming a spatial neighborhood in the antigen-antibody $(\mathrm{Ag}-\mathrm{Ab})$ complex. Identifying epitopes through experiments is tedious and expensive work, and therefore, there is a high risk of failure. Current progress in bioinformatics makes it possible to create vaccine designs through 3D visualization of protein antigen. Many characteristics, including composition, cooperativeness, hydrophobicity, and secondary structure, are considered in identifying potential substances for an epitope (Kringelum et al., 2013). Since no dominant characteristic helps experts to easily distinguish epitopes from other parts of the antigen, the risk of failure is quite high.

The availability of the 3D structure of the $\mathrm{Ag}-\mathrm{Ab}$ complex in the public domain and computational resources eases the development of predictive models using various methods, including the structure and sequence-based approaches. However, the conformational epitope prediction is still challenging. The structure-based approach can be divided into three including, dominant-characteristic-based, graph-based, and learningbased categories.

There are several characteristic-based approaches, including (1) CEP, which uses solventaccessibility properties, (2) Discotope using both solvent-accessibility-based properties and epitope log odds ratio of amino acid, (3) PEPITO that adds half-sphere exposure (HSE) to log odds ratio of amino acid in Discotope, (4) Discotope 2.0, which is an improved version of Discotope. It defines the log odd ratios in spatial contexts and adds half-sphere exposure (HSE) as a feature, and (5) SEPPA, which utilizes exposed and adjacent residual characteristics to form a triangle unit patch (Kulkarni-Kale et al., 2005; Andersen et al., 2006; Kringelum et al., 2012; Sun et al., 2009). The dominant-characteristic-based approach is limited by the number of features and the linear relationships between them.

Graph-based is yet another critical method, though only two from the same study were found during the literature review. Zhao et al. (2012) developed a subgraph that could represent the planar nature of the epitope. Although the model is designed to identify a single epitope, it can also detect multiples. Zhao et al. (2014) used features extracted from both antigens and the $\mathrm{Ag}-\mathrm{Ab}$ interaction, which is expressed by a coupling graph and later transformed into a general graph. 
75 The learning-based approach utilizes machine-learning to work with a large number of 76 features. It also uses nonlinear relationships between features to optimize model 77 performance. Rubinstein et al. (2009) used two Naïve Bayesian classifiers to develop

78

79

80

81

82

83

84

85

86

87

88

89

90

91

92

93

94

95

96

97

98

99

100

101

102

103

104

105

106

107

108

109

110

111

112 structure-based and sequence-based approaches. SEPPA 2.0 combines amino acid index (AAindex) characteristics in the SEPPA algorithm in the calculation of cluster coefficients (Qi et al., 2014; Kawashima et al., 2007). Aaindex in SEPPA 2.0 is consolidated via Artificial Neural Networks (ANN). However, SEPPA 3.0 adds the glycosylation triangles and glycosylation-related AAindex to SEPPA 2.0 (Chou et al., 2019). Glycosylation-related AAindex is consolidated to SEPPA 3.0 via ANN. Several researchers utilized the advantages of random forest (Dalkas and Rooman, 2017; Jespersen et al., 2017; Ren et al., 2014; Zhang et al., 2011). The main challenge in developing a conformational B cell epitope prediction model is the class imbalance problem. This is a condition where the sample of the target or epitope class is less than that of the nontarget or the non-epitope classes.

Several methods have been proposed to handle the class imbalance problem. However, studies that focus on handling this issue in epitope prediction models are still limited. Ren et al. (2014) and Zhang et al. (2011) used simple random undersampling to handle the class imbalance problem. Dalkas and Rooman (2017) used the Support Vector Machine (SVM) Synthetic Minority Over-sampling Technique (SMOTE) method, which is a variant of SMOTE. Another common approach used is weighted SVM, which is included in the cost-sensitive algorithm level category (Ren et al., 2015). Additionally, Zhang et al. (2014) used a costsensitive ensemble approach and proved that the method was superior to Easy Ensemble, Balance Cascade and SMOTEBoost (Liu et al., 2009; Chawla et al., 2003).

Currently, several studies focus on Class imbalance using various approaches that are mainly divided into four, including data and algorithm levels, cost-sensitive, and ensemble (Galar et al., 2012). In the data level approach, the resampling method is used to ensure a balanced distribution of data (Gary, 2013). The approaches under this category include undersampling, oversampling and a combination of both (Estrabrooks et al., 2004; Chawla et al., 2002; Chawla et al., 2008). In the algorithm level, the minority class is specifically considered. Most algorithms are equipped with a search system to identify rare patterns (Gary, 2013). The learning process of classifiers usually ignores the minority class. Specific recognition algorithms are used to detect rare patterns, providing different misclassification weights between minority and majority classes or different weights (Elkan, 2001; Batuwita and Palade, 2012; Japcowicz et al., 1995; Raskutti and Kowalczyk, 2003). In general, adding cost to an instance is categorized as cost-sensitive in the data level (Galar et al., 2012). The approach is also applied in the ensemble method (Blaszczynski and Stefanowski, 2014). However, the determination of the weight is carried out through trial and error.

The most common ensemble methods used to handle the class imbalance problem include bagging and boosting. In bagging, a balanced class sample is generated using the 
113 bootstrapping mechanism. The sampling methods used in this case include random 114 undersampling and oversampling, as well as SMOTE (Blaszczynski and Stefanowski, 2014; 115 Galar et al., 2012). In boosting, samples are selected iteratively and their weight calculated 116 based on the misclassification costs. Many boosting variations have been proposed, though 117 the most influential is the AdaBoost (Freund and Schapire, 1996).

118 Random oversampling and undersampling are the simplest sampling methods used in 119 balancing data distribution. Handling class imbalance in the preprocessing data is versatile 120 since it does not depend on the classifier used. Similarly, the random oversampling method 121 is versatile because it does not rely on the classifier used. However, its main drawback is 122 overfitting because new sample data are not added. The SMOTE technique avoids overfitting

123

124

125

126

127

128

129

130

131

132

133

134

135

136

137

138

139

140

141

142

143

144

145

146

147

148

149

150 by interpolating adjacent members of the minority class to create new sample data (Chawla et al., 2002). Furthermore, oversampling that considers certain conditions, such as the density distribution and the position of the sample point to the majority class, improves the performance of the classifier (He et al., 2008; Han et al., 2005). Random undersampling is a concern in the sense that loss of information from the dataset might occur. This is because of pruning may considerably affect and reduce its performance. To reduce the loss of information, several cluster-based methods have been used in resampling (Yen and Lee, 2009; Das et al., 2013; Sowah et al., 2016; Tsai et al., 2019).

Cluster-based undersampling can be conducted by omitting class labels (Yen and Lee, 2009; Das et al., 2013). Alternatively, it can be performed only on the negative class (Sowah et al., 2016; Lin et al., 2017; Tsai et al., 2019). Das et al. (2013) discarded the negative class data that overlap the positive in a specific cluster based on the degree of overlapping. According to Yen and Lee (2009), the samples from the negative class are proportional to the ones in the positive class in a particular cluster. Also, Sowah et al. (2016) randomly selected several sample data from each cluster. In Tsai et al. (2019), the cluster members were selected using optimization algorithms. Clustering samples of the negative to positive class may lead to a suboptimal cluster number of data in the negative class (Lin et al. 2017).

In this research, the cluster-based undersampling method is combined with SMOTE to obtain a balanced dataset. The parameter $r$ is defined to determine the proportion of the majority class data sampled and compared with the minority. A classifier model is built with the decision tree (DT) and SVM algorithms to assess the performance of the proposed method.

\section{Materials and Methods}

\section{Dataset}

This research uses Rubinstein's dataset as training (Rubinstein et al., 2009). The formation criteria of the training dataset are explained by Rubinstein et al. (2008). The study shows the following, (1) The Ag-Ab complex structure should contain antibodies with both heavy and light chains, (2) Contact between antigens and antibodies must occur in the complementarity-determining regions, (3) The amount of antigen residues binds to 
151

152

153

154

155

156

157

158

159

160

161

162

163

164

165

166

167

168

169

170

171

172

173

174

175

176

177

178

179

180

181

182

183

184

185

186

187

188

189

190

antibodies is large, and (4) The complex used cannot be similar to other complexes, as stated in the Structural Classification of Proteins criteria (Murzin et al., 1995). The training dataset consists of 76 antigen chains derived from 62 3D structure Ag-Ab complexes. The chain list is shown in Supplement Table 1. The complexes are downloaded from the Protein Data Bank (PDB) (Berman et al., 2000).

Two independent test sets are used, including Kringelum and SEPPA 3.0 (Kringelum et al., 2012; Chou et al., 2019). Kringelum's test set consists of 39 antigen chains. Data were filtered from 52 antigen chains and thirteen antigens were excluded from the list because they were used as training data with the compared method. The data released include 1AFV, 1BGX, 1RVF, 2XTJ, 3FMG, 3G6J, 3GRW, 3H42, 3MJ9, 3RHW, 3RI5, 3RIA, and 3RIF. The details of Zhang's test set are presented in Supplement Table 2a. The test set represents protein antigen in the general category. It is used to compare the CluSMOTE DT with the Discotope 1.2, Ellipro, Epitopia, EPCES, PEPITO and Discotope 2 (Andersen et al., 2006; Ponomarenko et al., 2009; Rubinstein et al., 2009; Liang et al., 2009; Sweredoski and Baldi, 2008; Kringelum et al., 2012). The SEPPA 3.0 test set is a glycoprotein category test set. This dataset consists of 98 antigen chains and eight were excluded because they were multiple epitopes, including 5KEM A1, 5KEM A2, 5T3X G1, 5T3X G2, 5TLJ X1, 5TLJ X2, 5TLK X1, and 5TLK X2. The test set was used to compare the CluSMOTE DT with the SEPPA 2.0 SEPPA 3.0, PEPITO, Epitopia, Discotope 2, CBTOPE and BepiPred 2.0 methods (Qi et al., 2014; Anshari and Raghava, 2010; Jespersen et al., 2017). The antigen list for the test set is presented in Supplement Table 2b.

\section{Conformational B Cell Epitope Prediction Method}

Conformational epitopes are residues of exposed antigens that are spatially close, though they form separate segments when viewed from sequences (Andersen et al., 2006). To build a conformational epitope prediction model, the steps needed include (1) preparing the dataset, (2) balancing the dataset, and (3) creating a classification model for the prediction of residual potential epitopes. The preparation step aims to build the training and testing datasets. The number of exposed residues considered as epitopes is less than the exposed residues that are not-epitopes. Balancing the dataset is meant to overcome the class imbalance found in Step 1, while the classification model categorizes residues as members of the epitope or non-epitope class.

\section{Data Preprocessing}

The creation of feature vectors and epitope annotations for the training and testing data is conducted on surface residues only. Relatively accessible surface area (RSA) is used as a parameter to distinguish surface and buried residues. Different values were used as limits, including the 0.05, 0.01, 0.1, and 0.25 thresholds (Rubinstein et al., 2009, Zhang et al., 2011; Kringelum et al., 2012; Ren et al., 2014; Dalkas et al., 2017). This variation affects the imbalance ratio between the data epitope and non-epitope classes. Although the standard burial and non-burial threshold are 0.05 , the value of 0.01 is used as the limit. This is because of the larger the surface exposure threshold, the smaller the predictive performance (Basu et al., 2011; Kringelum et al., 2012). Choosing 0.01 as the limit is relevant to the finding of 
191 Zheng et al. (2015), where all RSA values of epitopes are positive, though slightly larger than 192 zero.

193 The feature vectors used include accessible surface area (ASA), RSA, depth index (DI), protrusion index (PI), contact number (CN), HSE, quadrant sphere exposure (QSE), AAindex, $\mathrm{B}$ factor, and log odds ratio, as shown in Table 1.

196

197

198

199

200

201

202

203

204

205

206

207

208

209

210

211

212

213

214

215

216

217

218

219

220

221

222

223

224

225

226

227

ASA and RSA are the key features in determining if a residue is likely to bind to other molecules for accessibility reasons. Although several programs can be used to calculate ASA, the most commonly used include NACCESS and DSSP(Hubbard and Thornton, 1993; Kabasch and Sander, 1983). DSSP only calculates the total ASA per residue, while NACCESS computes the backbone, side chain, polar, and nonpolar ASA. However, NACCESS can only count one molecular structure at a time. These users need to create additional scripts to count several molecular structures at a time (Mihel et al., 2008). This study used the PSAIA application was used (Mihel et al., 2008). The PSAIA is not only limited to counting one molecular structure but can be used to calculate other features, including RSA, PI, and DI. No significant difference is observed between the ASA calculation results using NACCESS and PSAIA. The ASA attribute values used include the backbone, side chain, polar (including oxygen, nitrogen, and phosphorus), and nonpolar atoms (carbon atoms).

RSA is the result of the ASA value with the maximum figure calculated based on the GXG tripeptide theory, where G is glycine and X is the residual sought (Lee and Richard, 1971). The maximum value of ASA is obtained from Tien et al. (2013), which is an improvement of Rose and Sander (1994) and Miller et al. (1987). There was no difference in the list of datasets obtained using the three methods, as presented in Appendix 1. The RSA attribute values used include the total RSA of all atoms, backbone atoms, side-chain atoms, polar atoms (including oxygen, nitrogen, and phosphorus), and nonpolar atoms (carbon atoms).

DI: The DI of the $i$ th atom refers to its minimum distance to the exposed atoms. The DI attribute values used include the average and standard deviation of all atoms, average of side-chain atoms, maximum, and minimum.

PI: The PI is the ratio of the space of a sphere with a radius of $10 \mathrm{~A}$ centered on C $\alpha$ divided by the area occupied by the heavy atoms constituting proteins (Pintar, 2002). In this study, PI was calculated using the PSAIA software (Mihel et al., 2008). The PI attribute values used include the average and standard deviation of all atoms, average of side-chain atoms, maximum, and minimum.

CN, HSE, QSE: The CN is the total number of $\mathrm{C} \alpha$ adjacent to the residue measured under the microsphere environment. It is limited by a ball with radius $r$ centered on $\mathrm{C} \alpha$ (Nishikawa and Ooi, 1980). In HSE, the number of $\mathrm{C} \alpha$ was distributed in two areas, including the upper hemisphere and the lower hemisphere balls (Hamelryck, 2005). In QSE, the number of C $\alpha$ was specifically distributed in eight regions in the microsphere environment (Li et al., 2011). 
228

229

230

231

232

233

234

235

236

237

238

239

240

241

242

243

244

245

246

247

248

249

250

251

252

253

254

255

256

257

258

259

260

261

262

263

264

AAindex: The AAindex consists of 544 indices representing the amino acid physicochemical and biochemical properties (Kawashima et al., 2008). The AAindex value of each residue was extracted from component I of the HDRATJCI constituents in the aaindex1.txt file. The detail of component I of aaindex file is attached as a Supplement in Table 3.

$B$ factor: The $B$ factor indicates the flexibility of an atom or a residue. An exposed residue has a larger $B$ factor than a latent residue. The $B$ factor for each atom is derived from the PDB data. The attribute values used include the B factor of $\mathrm{C} \alpha$ and the average of all atoms or residues (Ren et al., 2014).

Log odds ratio: This feature is extracted based on the primary protein structure and calculated based on Andersen et al. (2006). A sliding window of size 9 residues was run in each sequence of antigens in the dataset to form overlapping segments that can be used in the calculation of the appearance of the individual residues. Each segment was grouped as an epitope or non-epitope depending on its center. The log odds ratio was calculated at the fifth position residue based on Nielsen et al. (2004). In this study, a segment would be included in the calculation in case the fifth position residue is exposed.

Epitope annotation on the antigen residue is carried out by analyzing the interaction in the PSAIA software (Mihel, 2008) using contact criterion, threshold, and Van der Waals radii. The maximum distance of 4 was derived from the chotia.radii file. The ASA change parameters include the Delta ASA, Z_Slice Size, and Probe Radius with values 1.0, 0.25, and 1.4 , respectively. The interaction analyzer output is a list of all adjacent residual pairs within the allowable distance range. A procedure for selecting antigen residues that bind to antibodies is created to obtain a list of epitopes.

\section{Handling Class Imbalance with CluSMOTE}

Resampling with undersampling and oversampling has advantages and disadvantages. Therefore, cluster-based sampling was conducted to minimize the loss of information caused by the pruning effect of undersampling. Oversampling with SMOTE has often proven to be reliable. Merging the two increases classifier performance. A parameter stating the degree of oversampling is used to identify the optimal combination.

This study proposed CluSMOTE, a cluster-based undersampling mechanism combined with SMOTE as shown in Fig. 2. Negative class data are clustered using the Hierarchical DensityBased Spatial Clustering of Applications with Noise (HDBSCAN) algorithm. This is meant to identify the optimal clusters based on stability (Campello et al., 2013). The number of clusters is less than the positive class data. This means each cluster contains several data. The simplest sampling mechanism is random selection. To select data, the cluster size and degree of oversampling should be considered.

The proposed CluSMOTE method uses the following steps,
1. Separate the positive and the negative class data. 
265

266

267

268

269

270

271

272

273

274

275

276

277

278

279

280

281

282

283

284

285

286

287

288

289

290

291

292

293

294

295

296

297

298

299

300

2. Cluster the negative class (-) using the HDBScan algorithm.

3. Take a certain number of data items from each cluster. Consider the ratio of the number of clusters to the overall members of the negative class. The samples from the Ci cluster is defined in Eq. (1), according to Sowah et al. (2016).

$$
\text { Size_Ci }=r \times M I \times M_{-} c i / M A \text {, }
$$

where $\mathrm{MI}$ is the number of minority class samples, $\mathrm{MA}$ is the total number of majority classes, M_ci is the number of $\mathrm{Ci}$ cluster members, and $\mathrm{r}$ is the negative class dataset ratio from the cluster. In case $r=2$, the number of negative class datasets to be formed is twice the positive class datasets. The samples are taken from each cluster randomly.

4. Combine positive classes with all datasets taken in Step 3.

5. Carry out SMOTE on the results obtained in Step 4.

Program implementation was conducted in the Java programming environment with NetBeans IDE 8.2. A new class for implementing the CluSMOTE method was implemented in the Java Language Programming, supported by the JSAT statistics library version 0.09 (Raff, 2017).

\section{Classification Algorithm}

Two classification algorithms, SVM and DT, were used to evaluate the performance of CluSMOTE. Generally, SVM is a popular learning algorithm used in previous studies of conformational epitope prediction. DT is often used to handle the class imbalance problem and classified as one of the top 10 data mining algorithms (Galar, 2012).

This study uses the JSAT (Raff, 2017) software package, utilizing the Pegasos SVM with a mini-batch linear kernel (Shalev-Shwartz et al., 2000). Pegasos SVM works fast since the primal update process is carried out directly, and no support vector is stored. The default values used for the epoch, regularization, and batch size parameters include $5,1 \mathrm{e}-4$, and 1 , respectively. The decision tree is formed by nodes that are built on the principle of decision stump (Iba and Langley, 1992). Also, the study used a bottom-up pessimistic pruning with error-based pruning from the C4.5 algorithm (Quinlan, 1993). The proportion of the data set used for pruning is 0.1 .

\section{Performance Measurement of the Conformational Epitope Prediction Model}

A dataset used for conformational epitope prediction contains the class imbalance problem. The area used is mainly under the ROC curve (AUC) as a performance parameter. In class imbalance, the AUC is a better measure than accuracy, which is biased to the majority class. Another performance parameter used is F-measure, as expressed in Eq. (2):

$$
F_{m}=\frac{2 * P P V^{*} S E}{P P P+S E}=2 * T P /(2 * T P+F N+F P),
$$


302

303

304

305

306

307

308

309

310

311

312

313

314

315

316

317

318

319

320

321

322

323

324

325

326

327

328

329

330

331

332

333

334

where PPV $=\mathrm{TP} /(\mathrm{TP}+\mathrm{FP})$ and SE denote sensitivity or TPR. The F-measure is not affected by imbalance conditions provided the training data used are balanced (Batuwita and Palade, 2009). Other metrics that can be used to assess performance include Gmean and adjusted Gmean (AGm). The Gmean is expressed in Eq. (3) below,

$$
\text { Gmean }=\sqrt{S P^{*} S E},
$$

where SP denotes specificity/TPR and SE denotes sensitivity/FPR. AGm is expressed in Eq. (4):

$$
A G m=\left\{\begin{array}{ccc}
\left(G m+S P^{*} N\right) /\left(1+N_{n}\right) & \text { if } & S E>0 \\
0 & \text { if } & S E=0
\end{array}\right.
$$

where Gm is Gmean, SP specificity, SE sensitivity, and Nn the proportion of negative samples in the dataset. AGm is suitable for application in case an increase in TPR is achieved with minimal reduction in TNR. Generally, this criterion is suitable for bioinformatics cases, where errors in the identification of negative classes are unexpected (Batuwita and Palade, 2009). In the case of epitope prediction, the false negative is not expected to be high. The selection of the wrong residue leads to the failure of the subsequent process.

\section{Results and Discussions}

The complex-based leave-one-out cross-validation method is used to test the reliability of the classifier model. Each training set is built from the $n-1$ complexes and tested with a test set from the $n$-th complex. Model performance was measured using seven parameters, including TPR, TNR, precision, AUC, Gmean, AGm, and F-measure.

\section{Effect of the Selection of the $r$ Value on Model Performance}

In the original dataset, the ratio of imbalance between negative and positive classes is 10:1. To assess the effectiveness of sampling, this study utilized several $r$ values derived using the ratio of negative to positive class data. The value $r=1$ indicates that only the clustering and undersampling steps are applied. The value $r=2$ indicates that the number of negative class datasets is twice the number of positive ones. The test results obtained without a balancing mechanism show the effectiveness of the proposed resampling method. The results of the assessment of the performance of the classification model expressed by the TPR, TNR, precision, AUC, Gmean, AGm, and F-measure parameters are shown in Table 2.

The results of internal model validation on several variations of the $r$-value are also shown in Table 2. Where the $r$-value varies from $r=1$ to $r=5$, both in CluSMOTE DT and CluSMOTE SVM, the TPR and the FPR value tends to decrease with the increase in the $r$-value. The larger the degree of oversampling, the smaller the TPR. The TNR value, as well as precision, also tends to increase with the increase in the $r$-value. The increase in TNR values means more 
335

336

337

338

339

340

341

342

343

344

345

346

347

348

349

350

351

352

353

354

355

356

357

358

359

360

361

362

363

364

365

366

367

368

369

370

371

372

negative classes are recognized. This can also be interpreted as TNR value increases means less information loss of the negative class. These two conditions indicate a trade-off between the degrees of oversampling and undersampling. Oversampling without undersampling yields TNR and precision values greater than undersampling without oversampling. Similarly, undersampling without oversampling yields TPR and FPR values greater than oversampling without undersampling. This finding indicates the undersampling mechanism is more effective in increasing positive class recognition than the oversampling, which is consistent with previous studies. Also, the resampling mechanism increases the TPR and FPR values compared to no resampling. However, the overall performance improvement indicated by the AUC, Gmean, AGm, and F-measure is not significant.

In CluSMOTE DT, AUC and Gmean have the same tendency. The best AUC and Gmean are 0.815 and 0.811 at $r=2$ respectively. The AGm and F-measure values also have the same tendency, though the values are different. In DT, the best AGm and F-measure are obtained using the SMOTE oversampling method. In the SVM classifier, the best AGm is obtained using the SMOTE oversampling mechanism. However, the best F-measure is obtained using CluSMOTE at $r=5$.

Previous studies on class imbalance stated that the hybrid resampling method could significantly improve performance. However, this was not the case in epitope prediction using the CluSMOTE DT method. No $r$ value significantly influenced the overall performance improvement expressed by the AUC, Gmean, AGm, and F-measure. In case the TPR and TNR values are considered together, the selection of $r=2$ is quite good as shown by the AUC and Gmean values. The selection of $r$ values based on the experiment shows opposing conditions between the TPR and TNR. From Table 2, the best performance using AUC and Gmean is fairer compared to Agm and F-score. In the best AUC and AGm, a balanced proportion was obtained between the TPR and TNR. The best AGm and F-score resulted from the lowest TPR value. Generally, the performance models built with DT exhibit better performance than those from SVM. The performance of SVM is likely to be affected by kernel selection problems. Linear kernels are cannot separate the classes in polynomial cases. Other configurations or models may be explored for future work.

\section{Comparison of the Proposed Method with Previous Methods}

CluSMOTE DT was evaluated on an independent test set from Kringelum et al. (2012) by filtering the dataset from the details used in the training process of the method being compared. A total of 39 antigen data were used in the comparison, as listed in Table 4 . The final results of the test show that CluSMOTE with $r=2$ is superior to the other methods with an average AUC value of 0.83 . The average AUC values of Discotope, Ellipro, Epitopia, EPCES, PEPITO, and Discotope 2.0 were $0.727,0.721,0.673,0.697,0.746$, and 0.744 , respectively.

CluSMOTE DT with $r=2$ was evaluated on the independent test set of glycoprotein antigen by Zhou et al. (2019). Testing with glycoprotein antigen showed that the performance of 
373 CluSMOTE DT was similar to that of SEPPA 3.0, with the AUC values of 0.766 and 0.739 , 374 respectively. Both CluSMOTE DT and SEPPA 3.0 were superior to Epitopia, Discotope 2.0, 375 PEPITO, CBTOPE, SEPPA 2.0, and BepiPred 2.0. The detailed performance of the eight

376

377

378

379

380

381

382

383

384

385

386

387

388

389

390

391

392

393

394

395

396

397

398

399

400

401

402

403

404

405

406

407

408

409

410 methods compared is shown in Table 5. The AUC achieved by CluSMOTE DT is comparable to the one from SEPPA 3.0, showing that the proposed method might handle epitope cases with glycoprotein well. The model developed with CluSMOTE uses the dataset presented by Andersen et al. (2006), which consists of 76 antigen structures. The number of complex structures used in the CluSMOTE model is less than that used in SEPPA 3.0, which consists of 767 antigen structures. The small number of antigen structures speeds up the training time for model development.

\section{Conclusions}

An epitope is a small part of the exposed antigen that creates class imbalance problems in the prediction of learning-based conformational epitopes. In this study, the CluSMOTE method was proposed to overcome the class imbalance problem in the prediction of the conformational epitope. The study shows that CluSMOTE considerably increases the TPR compared to SMOTE only. The comparison of the proposed model with state-of-the-art methods in the two datasets shows that CluSMOTE DT is comparable to or better than other methods. Its mean AUC values in Kringelum and the SEPPA 3.0 test sets are 0.83 and 0.766, respectively. This result shows that CluSMOTE DT is better than other methods in classifying the general protein antigen, though it is comparable to SEPPA 3.0 in the glycoprotein antigen.

\section{Acknowledgments}

The authors thank the Publisher and Publication Agency of Universitas Gadjah Mada for the English proof-reading of this manuscript.

\section{References}

Andersen PH, Nielsen M, Lund OLE. Prediction of residues in discontinuous B-cell epitopes using protein 3D structures. Protein Sci. 2006;15:2558-2567. doi:10.1110/ps.062405906.2558

Ansari HR, Raghava GPS. Identification of conformational B-cell Epitopes in an antigen from its primary sequence. Immunome Res. 2010;6(1):6. doi:10.1186/1745-7580-6-6

Basu S, Bhattacharyya D, Banerjee R. Mapping the distribution of packing topologies within protein interiors shows predominant preference for specific packing motifs. BMC Bioinformatics. 2011;12(195).

Batuwita R, Palade V. Class Imbalance Learning Methods For Support Vector. In: He H, Ma Y, eds. Imbalanced Learning: Foundations, Algorithms, and Applications. John Wiley \& Sons, Inc; 2012:1-20.

Berman HM, Westbrook J, Feng Z, Gilliland G, Bhat TN, Weissig H, Shindyalov IN, Bourne PE. The Protein Data Bank. Nucleic Acids Res. 2000;28(1):235-242.

Blaszczynski J, Stefanowski J. Neighbourhood sampling in bagging for imbalanced data. Neurocomputing. 2014. doi:10.1016/j.neucom.2014.07.064 
411 Campello RJGB, Moulavi D, Sander J. Density-Based Clustering Based on Hierarchical Density 412 Estimates. In: Pei J, Tseng VS, Cao L, Motoda H, Xu G, eds. Advances in Knowledge Discovery and Data 413 Mining PAKDD Part II LNAI. Springer, Berlin, Heidelberg; 2013:160-172.

414 Chawla N V, Cieslak DA, Hall LO, Joshi A. Automatically countering imbalance and its empirical 415 relationship to cost. Data Min Knowl Discov. 2008;17(2):225-252. doi:10.1007/s10618-008-0087-0 416 Chawla N V, Bowyer KW, Hall LO, Kegelmeyer WP. SMOTE: Synthetic Minority Over-sampling 417 Technique. J Artif Intell Res. 2002;16:321-357.

418 Chawla N V, Lazarevic A, Hall LO, Bowyer KW. SMOTEBoost: Improving Prediction of the Minority 419 Class in Boosting 7th European Conference on Principles and Practice of Knowledge Discovery 420 SMOTEBoost : Improving Prediction of the Minority Class in Boosting. In: Lecture Notes in Computer 421 Science; 2003. doi:10.1007/978-3-540-39804-2

422 Dalkas GA, Rooman M. SEPIa, a knowledge-driven algorithm for predicting conformational B-cell 423 epitopes from the amino acid sequence. BMC Bioinformatics. 2017;18(95):1-12. 424 doi:10.1186/s12859-017-1528-9

425 Das B, Krishnan NC, Cook DJ. Handling Class Overlap and Imbalance to Detect Prompt Situations in 426 Smart Homes. In: IEEE 13th International Conference on Data Mining Workshops. IEEE Computer 427 Society; 2013:266-273. doi:DOI 10.1109/ICDMW.2013.18

428 Drummond C, Holte RC. C4. 5 , Class Imbalance, and Cost Sensitivity: Why Under-Sampling beats 429 Over-Sampling. In: ICML Workshop on Learning from Imbalanced Data Sets II. Washington DC; 2003.

430 Elkan C. The Foundations of Cost-Sensitive Learning. In: Proceedings of the Seventeenth International 431 Joint Conference on Artificial Intelligence; 2001:973-978.

432 Estabrooks A, Jo T, Japcowick N. A Multiple Resampling Method for Learning from Imbalanced Data 433 Sets. Comput Intell. 2004;20(1):18-36.

434 Freund Y, Schapire RE. Experiments with a New Boosting Algorithm. In: Machine Learning: 435 Proceedings Of the Thirteenth International Conference; 1996.

436 Galar M, Fern A, Barrenechea E, Bustince H. Hybrid-Based Approaches. IEEE Trans Syst Cybern 437 PART C Appl Rev. 2012;42(4):463-484.

438 Gary MW. Foundation of Imbalanced Learning. In: He H, Ma Y, eds. Imbalanced Learning: 439 Foundations, Algorithms, and Applications. John Wiley \& Sons, Inc; 2012:13-42

440 Hamelryck T. An Amino Acid Has Two Sides : A New 2D Measure Provides a Different View of Solvent 441 Exposure. ProteinsStructure, Funct Bioinforma. 2005;59(September 2004):38-48. 442 doi:10.1002/prot.20379

443 Hubbard SJ, Thornton JM. NACCESS. Department of Biochemistry and Molecular Biology, University 444 College London; 1993.

445 Iba, W and Langley, P. Induction of One-Level Decision Trees, in ML92: Proceedings of the Ninth 446 International Conference on Machine Learning, Aberdeen, Scotland, 1-3 July 1992, San Francisco, CA: 447 Morgan Kaufmann, 1992(July): pp. 233-240.

448 Japkowicz N, Myers C, Gluck M. A Novelt y Detectio n Approac h t o Classificatio. In: The Fourteenth 449 Joint Conference on Artificial Intelligence; 1989:518-523.

450 Jespersen MC, Peters B, Nielsen M, Marcatili P. epitope prediction using conformational epitopes. 451 Nucleic Acids Res. 2017;45(May):24-29. doi:10.1093/nar/gkx346

452 Kabsch W, Sander C. Dictionary of Protein Secondary Structure:Pattern Recognition of Hydrogen453 Bonded and Geometrical Features. Biopolymers. 1983;22:2577-2637. 
454 Kawashima S, Pokarowski P, Pokarowska M, Kolinski A, Katayama T, Kanehisa M. AAindex : 455 amino acid index database, progress report 2008. Nucleid Acids Res. 2008;36(November 2007):202456 205. doi:10.1093/nar/gkm998

457 Kringelum JV, Lundegaard C, Lund 0, Nielsen M. Reliable B Cell Epitope Predictions : Impacts of 458

459 Method Development and Improved Benchmarking. PLOS Comput Biol. 2012;8(12).

460 Kulkarni-kale U, Bhosle S, Kolaskar AS. CEP : a conformational epitope prediction server. Nucleic Acids Res. 2005;33(Web Server issue):168-171. doi:10.1093/nar/gki460

461

462 Lee B, Richards FM. The Interpretation of Protein Structures : Estimation of Static Accessibility. J Mol Biol. 1971;55:379-400.

464

Li P, Pok G, Ã KSJ, Shon HS, Ryu KH. QSE : A new 3-D solvent exposure measure for the analysis of 465 protein structure. Proteomics. 2011;11:3793-3801. doi:10.1002/pmic.201100189

466

Liang S, Zheng D, Zhang C, Zacharias M. consensus scoring. BMC Bioinformatics. 2009;10(302):110. doi:10.1186/1471-2105-10-302

467 Lin W, Tsai C, Hu Y, Jhang J. Clustering-based undersampling in class-imbalanced data. Inf Sci (Ny). 468 2017;409-410:17-26. doi:10.1016/j.ins.2017.05.008

469 Liu X, Wu J, Zhou Z. Exploratory Undersampling for. IEEE Trans Syst Cybern -PART B CYBERNETICS. 470 2009;39(2):539-550.

471 Mihel J, Šiki M, Tomi S, Jeren B, Vlahovi K. PSAIA-Protein Structure and Interaction Analyzer. BMC 472 Struct Biol. 2008;11:1-11. doi:10.1186/1472-6807-8-21

473 Millerl S, Janin J, Leskv AM, Chothial C, Laboratories CI, Physicochimique DB. Interior and Surface 474 of Monomeric Proteins t. J Mol Biol. 1987;196:641-656.

475 Murzin AG, Brenner SE, Hubbard T, Chothia C. SCOP : A Structural Classification of Proteins 476 Database for the Investigation of Sequences and Structures. J Mol Biol. 1995;247:536-540.

478 Nielsen M, Lundegaard C, Worning P. Improved prediction of MHC class I and class II epitopes using a novel Gibbs sampling approach. Bioinformatics. 2004;20(9):1388-1397. doi:10.1093/bioinformatics/bth100

480 Nishikawa K, Ooi T. Prediction of the surface-interior diagram of globular proteins by an empirical 481 method.pdf. Int J Pept Protein Res. 1980;16:19-32.

483 Bioinformatics. 2002;18(7):980-984.

484 Ponomarenko J, Bui H, Li W, Fusseder N, Bourne PE, Sette A, Peters B. ElliPro : a new structure485 based tool for the prediction of antibody epitopes. BMC Bioinformatics. 2008;9(514):1-8. 486 doi:10.1186/1471-2105-9-514

487 Qi T, Qiu T, Zhang Q, Tang K, Fan Y, Qiu J, Wu D, Zhang W, Chen Y, Gao J, Zhu R, Cao Z. SEPPA 2. 4880 -- more refined server to predict spatial epitope considering species of immune host and 489 subcellular localization of protein antigen. Nucleid Acids Res. 2014;42(May):59-63. 490 doi:10.1093/nar/gku395

491 Quinland JR, C4.5 programs for machine learning. San Mateo, CA: Morgan Kaufmann, 1993.

492 Raff E. JSAT : Java Statistical Analysis Tool, a Library for Machine Learning. J Mach Learn Res. 493 2017;18:1-5.

494 Raskutti B, Kowalczyk A. Extreme Re-balancing for SVMs: a case study. In: Workshop on Learning 495 from Imbalanced Datasets II. Washington DC; 2003. 
496 Ren J, Liu Q, Ellis J, Li J. Tertiary structure-based prediction of conformational B-cell epitopes

497

498

499

500

501

502

503

504

505

506

507

508

509

510

511

512

513

514

515

516

517

518

519

520

521

522

523

524

525

526

527

528

529

530

531

532

533

534

535

536 through B factors. Bioinformatics. 2014;30:264-273. doi:10.1093/bioinformatics/btu281

Ren J, Liu Q, Ellis J, Li J. Positive-unlabeled learning for the prediction of conformational B-cell epitopes. BMC Bioinformatics. 2015;16(Suppl 18):1-15.

Rost B, Sender C. Conservation and Prediction of Solvent Accesibility in Protein Families. ProteinsStructure, Funct Genet. 1994;20(November):216-226. doi:10.1002/prot.340200303

Rubinstein ND, Mayrose I, Halperin D, Yekutieli D, Gershoni JM, Pupko T. Computational characterization of B-cell epitopes. Mol Immunol. 2008;45:3477-3489. doi:10.1016/j.molimm.2007.10.016

Rubinstein ND, Mayrose I, Pupko T. A machine-learning approach for predicting B-cell epitopes. Mol Immunol. 2009;46:840-847. doi:10.1016/j.molimm.2008.09.009

Sowah RA, Agebure MA, Mills GA, Koumadi KM, Fiawoo SY. New cluster undersampling technique for class imbalance learning. Int J Mach Learn Comput. 2016;6(3):205-214. doi:10.18178/ijmlc.2016.6.3.599

Sun J, Wu D, Xu T, Wang X, Xu X, Tao L, Li, YX, Cao ZW. SEPPA : a computational server for spatial epitope prediction of protein antigens. Nucleic Acids Res. 2009;37:612-616. doi:10.1093/nar/gkp417

Sweredoski MJ, Baldi P. PEPITO : improved discontinuous B-cell epitope prediction using multiple distance thresholds and half sphere exposure. Bioinforma Appl Note. 2008;24(12):1459-1460. doi:10.1093/bioinformatics/btn199

Tien MZ, Meyer AG, Sydykova DK, Spielman SJ, Wilke CO. Maximum allowed solvent accessibilites of residues in proteins. PLoS One. 2013;8(11). doi:10.1371/journal.pone.0080635

Tsai C, Lin W, Hu Y, Yao G. Under-sampling class imbalanced datasets by combining clustering analysis and instance selection. Inf Sci (Ny). 2019;477:47-54.

Yen S, Lee Y. Expert Systems with Applications Cluster-based under-sampling approaches for imbalanced data distributions. Expert Syst Appl. 2009;36:5718-5727. doi:10.1016/j.eswa.2008.06.108

Zhang W, Xiong Y, Zhao M, Zou H, Ye X, Liu J. Prediction of conformational B-cell epitopes from 3D structures by random forests with a distance-based feature. BMC Bioinformatics. 2011;12(341).

Zhang J, Zhao X, Sun P, Gao B, Ma Z. Conformational B-cell epitopes prediction from sequences using cost-sensitive ensemble classifiers and spatial clustering. Biomed Res Int. 2014;2014.

Zheng W, Ruan J, Hu G, Wang K, Hanlon M, Gao J. Analysis of Conformational B-Cell Epitopes in the Antibody-Antigen Complex Using the Depth Function and the Convex Hull. PLoS One. 2015;10(8):116. doi:10.1371/journal.pone.0134835

Zhao L, Wong L, Lu L, Hoi SCH, Li J. B-cell epitope prediction through a graph model. BMC Bioinformatics. 2012;13(Sup 17)(S20):1:12.

Zhao L, Hoi SCH, Li Z, Wong L, Nguyen H, Li J. Coupling graphs, efficient algorithms and B-cell epitope prediction. IEEE/ACM Trans Comput Biol Bioinforma. 2014;11(1):7-16.

Zhou C, Chen Z, Zhang L, Zhang L, Yan D, Mao T, Tang K, Qiu T,Cao Z,. SEPPA 3 . 0 -- enhanced spatial epitope prediction enabling glycoprotein antigens. Nucleic Acids Res. 2019;47(May):388-394. doi:10.1093/nar/gkz413 
Figure 1

Development stage of conformational B-cell epitopes prediction

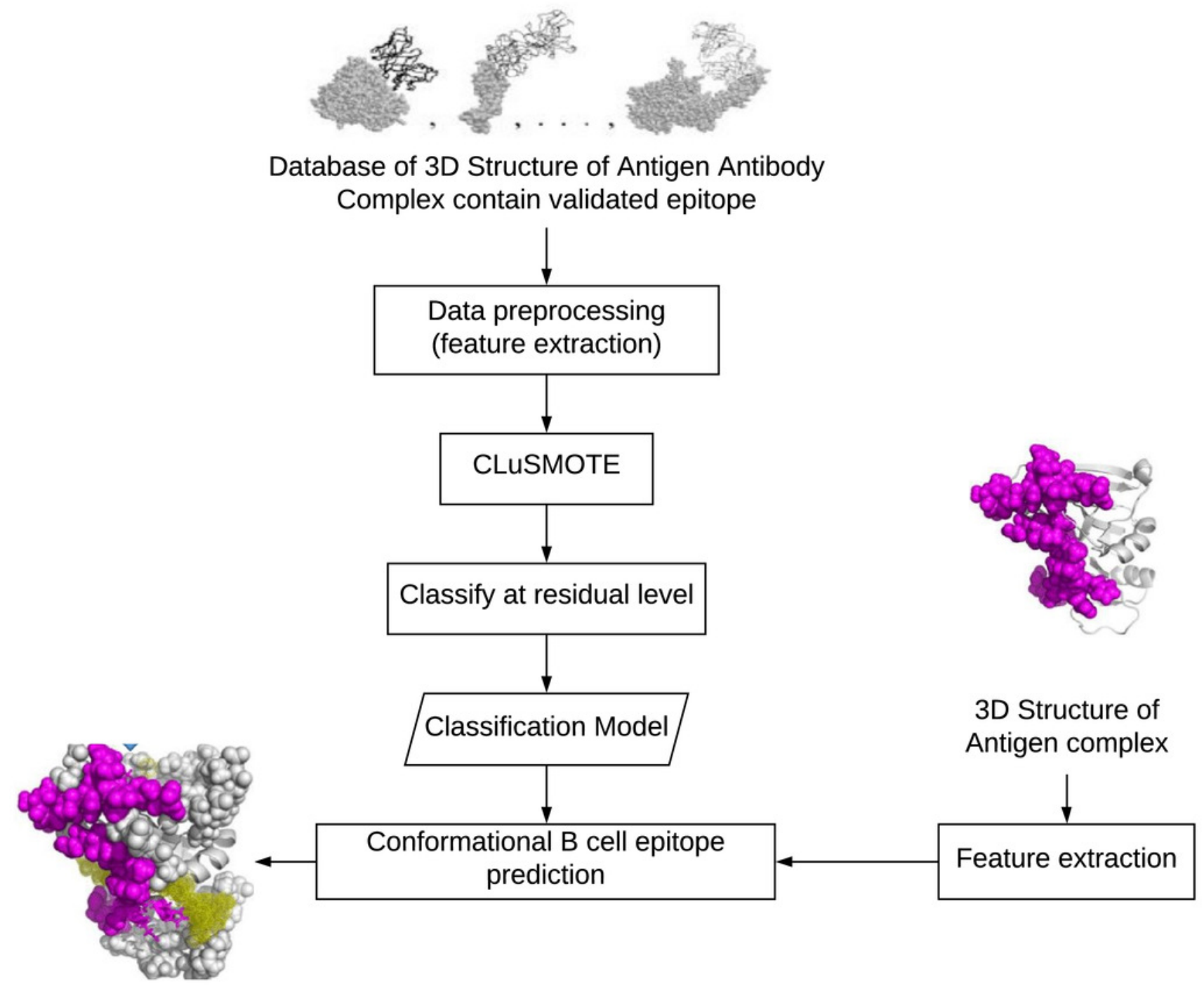

Epitope candidate 


\section{Figure 2}

\section{CluSMOTE sampling}

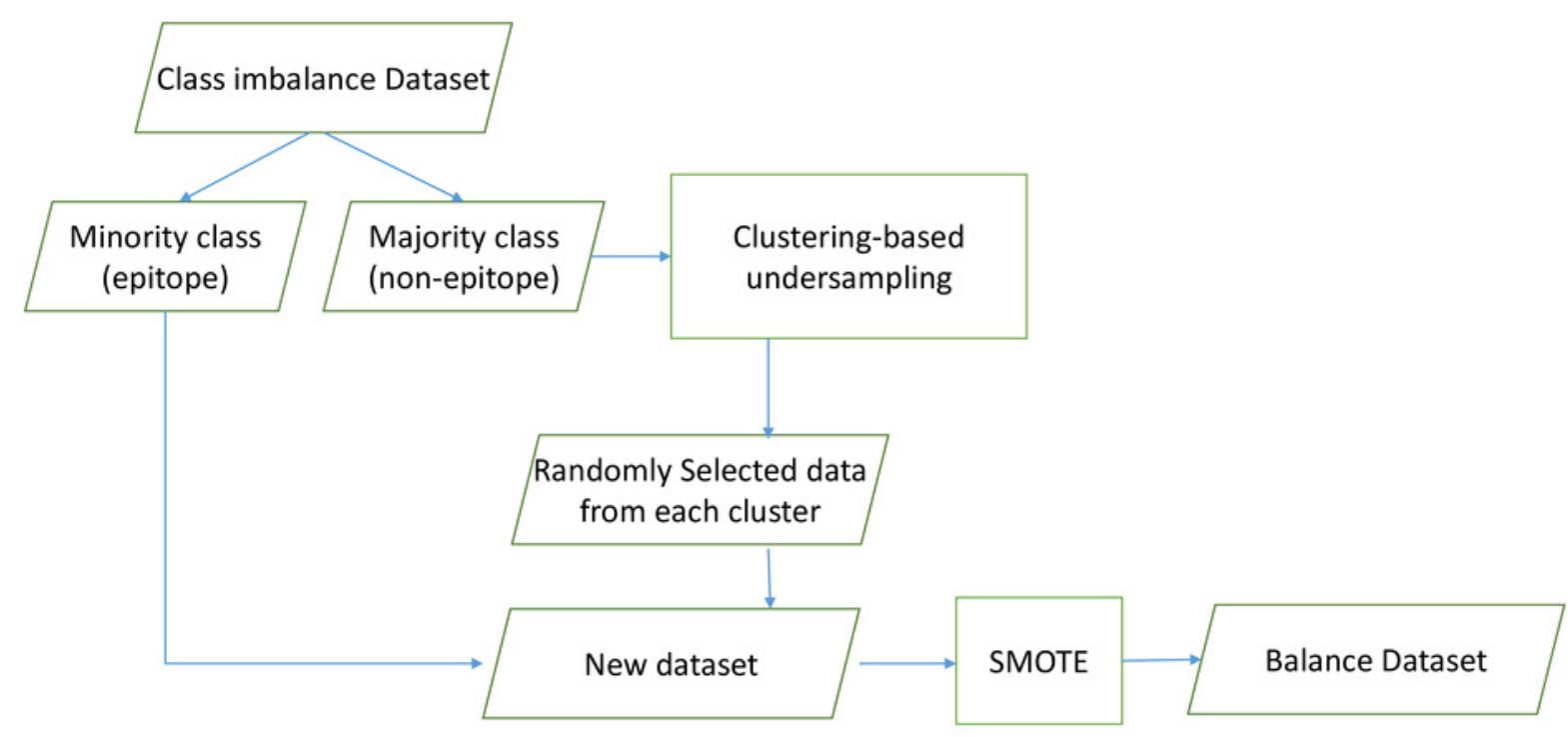




\section{Table $\mathbf{1}$ (on next page)}

Features for antigenic determinant and the methods to compute 
1 Table 1. Features for antigenic determinant and the methods to compute

\begin{tabular}{|l|l|l|}
\hline Category & Feature & Data Source/Method \\
\hline Structural & ASA & PSAIA (Mihel et al, 2008) \\
\hline & RSA & PSAIA (Mihel et al, 2008) \\
\hline & Protrusion Index & PSAIA (Mihel et al, 2008) \\
\hline & CN & Nishikawa and Ooi (1979) \\
\hline & HSE & Hamelryck, 2005 \\
\hline Physicochemical & QSE & Li et al,2011 \\
\hline & AAIndex & Kawashima, 2008 \\
\hline Statistic & B factor & Ren et al, 2014; Ren et al, 2015 \\
\hline & Log-odd ratio & Andersen et al, 2006 \\
\hline
\end{tabular}

2

3 ASA: solvent-accessible surface area

4 RSA: Relative solvent-accesible Surface Area

5 CN: Contact Number

6 HSE: Half-Sphere Exposure

7 QSE: Quadrant Sphere Exposure

8 AAIndex: Amino Acid Index 


\section{Table 2 (on next page)}

Performance of classification model with variations in the r-value 
Table 2. Performance of classification model with variations in the $r$ value

\begin{tabular}{|c|c|c|c|c|c|c|c|c|c|c|c|}
\hline No & Resampling Method & $r$ & classifier & $\begin{array}{l}\text { TPR } \\
\text { (recall) }\end{array}$ & TNR & $\begin{array}{l}\text { Precision } \\
\text { (PPV) }\end{array}$ & FPR & AUC & Gmean & $\begin{array}{l}\text { Adjusted } \\
\text { Gmean }\end{array}$ & Fmeasure \\
\hline 1 & Cluster-based only & 1 & DT & $0,855^{*}$ & 0,769 & 0,454 & $0,231^{*}$ & 0,812 & 0,806 & 0,791 & 0,581 \\
\hline 2 & CluSMOTE & 2 & DT & 0,797 & 0,834 & 0,526 & 0,163 & $0,815^{*}$ & $0,811^{*}$ & 0,823 & 0,622 \\
\hline 3 & CluSMOTE & 3 & DT & 0,764 & 0,862 & 0,558 & 0,138 & 0,813 & 0,807 & 0,833 & 0,634 \\
\hline 4 & CluSMOTE & 4 & DT & 0,730 & 0,881 & 0,575 & 0,119 & 0,806 & 0,796 & 0,835 & 0,631 \\
\hline 5 & CluSMOTE & 5 & DT & 0,724 & 0,880 & 0,591 & 0,120 & 0,802 & 0,794 & 0,834 & 0,641 \\
\hline 6 & SMOTE only & - & DT & 0,644 & $0,939^{*}$ & $0,732^{*}$ & 0,061 & 0,791 & 0,771 & $0,848^{*}$ & $0,675^{*}$ \\
\hline 7 & No Resampling & - & DT & 0,637 & 0,939 & 0,730 & 0,061 & 0,788 & 0,767 & 0,846 & 0,669 \\
\hline 8 & Cluster-based only & 1 & SVM & $0,591^{* *}$ & 0,668 & 0,393 & $0,328^{* *}$ & 0,629 & 0,579 & 0,620 & 0,388 \\
\hline 9 & CluSMOTE & 2 & SVM & 0,577 & 0,746 & 0,441 & 0,254 & $0,661^{\text {** }}$ & $0,600^{* *}$ & 0,666 & 0,400 \\
\hline 10 & CluSMOTE & 3 & SVM & 0,498 & 0,790 & 0,486 & 0,210 & 0,644 & 0,580 & 0,675 & 0,396 \\
\hline 11 & CluSMOTE & 4 & SVM & 0,475 & 0,801 & 0,508 & 0,199 & 0,638 & 0,566 & 0,672 & 0,387 \\
\hline 12 & CluSMOTE & 5 & SVM & 0,468 & 0,819 & 0,529 & 0,178 & 0,643 & 0,572 & 0,683 & $0,401^{* *}$ \\
\hline 13 & SMOTE only & - & SVM & 0,384 & $0,881^{* *}$ & $0,606^{* *}$ & 0,119 & 0,632 & 0,532 & 0,688 & 0,368 \\
\hline 14 & No Resampling & - & SVM & 0,409 & 0,874 & 0,569 & 0,126 & 0,641 & 0,557 & $0,699 * *$ & 0,392 \\
\hline
\end{tabular}

3 TPR: True Positive Rate

4 TNR: True Negaitive Rate

5 AUC: Area Under ROC Curve

6 Gmean: Geometric mean

7 *: the best parameter value in DT model

$8 * *:$ the best parameter vaue in SVM model 